\title{
Mechanisms of Interstitial Flow-Induced Remodeling of Fibroblast-Collagen Cultures
}

\author{
Chee Ping NG ${ }^{1}$ and Melody A. Swartz ${ }^{1,2}$ \\ ${ }^{1}$ Department of Chemical and Biological Engineering, Northwestern University, Evanston, IL 60208 and ${ }^{2}$ Institute of Bioengineering, \\ École Polytechnique Fédérale de Lausanne (EFPL), 1015 Lausanne, Switzerland
}

(Received 23 August 2005; accepted 11 November 2005; published online: 16 February 2006)

\begin{abstract}
Interstitial fluid flow, critical for macromolecular transport, was recently shown to drive fibroblast differentiation and perpendicular cell and matrix alignment in 3D collagen cultures. Here we explore the mechanisms underlying this flowinduced cell and collagen alignment. Cell and matrix alignment was assessed from 3D confocal reflectance stacks using a Fast Fourier Transform method. We found that human dermal and lung fibroblasts align perpendicular to flow in the range of 5$13 \mu \mathrm{m} / \mathrm{s}\left(0.1-0.3 \mathrm{dyn} / \mathrm{cm}^{2}\right)$ in collagen; however, neither cells nor matrix fibers align in fibrin cultures, which unlike collagen, is covalently cross-linked and generally degraded by cell fibrinolysis. We also found that even acellular collagen matrices align weakly upon exposure to flow. Matrix alignment begins within $12 \mathrm{~h}$ of flow onset and continues, along with cell alignment, over $48 \mathrm{~h}$. Together, these data suggest that interstitial flow first induces collagen fiber alignment, providing contact guidance for the cells to orient along the aligned matrix; later, the aligned cells further remodel and align their surrounding matrix fibers. These findings help elucidate the effects of interstitial flow on cells in matrices and have relevance physiologically in tissue remodeling and in tissue engineering applications.
\end{abstract}

Keywords-Fibrin, In vitro, Mechanobiology, Shear stress.

\section{INTRODUCTION}

Interstitial flow refers to the movement of fluid through the extracellular matrix (ECM) or interstitium of a tissue. It constitutes an important component of the microcirculation by providing convection necessary to transport large proteins through the interstitial space. There is abundant evidence that mechanical forces can help direct ECM organization, particularly related to mechanical properties and functionality. Fibroblasts, smooth muscle cells, osteoblasts, chondrocytes, and many other cell types reside within a 3D environment and are exposed to interstitial fluid forces. Thus, interstitial flow may play an important role in maintaining ECM properties via such cells.

Address correspondence to Melody A. Swartz, Institute of Bioengineering, Station 15, École Polytechnique Fédérale de Lausanne (EFPL), 1015 Lausanne, Switzerland. Electronic mail: melody.swartz@ epfl.ch
We previously showed that in response to low levels of interstitial fluid flow in 3D collagen culture, human fibroblasts align in the perpendicular direction to flow $^{23}$ and differentiate into myofibroblasts. ${ }^{22}$ Here we explore mechanisms behind this alignment, examining passive flow-induced alignment vs. active cell remodeling and reorganization. Either of the mechanisms is feasible. On one hand, if ECM fibers are reorganized directly by the flow, these fibers may then reorient the cells they support. Such contact guidance phenomena have been demonstrated in mechanically realigned matrices under non-flow conditions ${ }^{8,15,17,30,33}$ and have been applied to tissue engineering of anisotropic tissues such as arterial media ${ }^{1,15}$ and heart muscle. ${ }^{5}$ On the other hand, it is well established that fibroblasts can remodel their ECM and align during contraction and migration, ${ }^{11,13,29}$ and smooth muscle cells in 2D monolayers have been shown to align in the perpendicular direction to applied fluid shear stress. ${ }^{20}$ Finally, it is also possible that the flow-induced reorganization results from combined effects of both active and passive mechanisms, since interstitial flow can affect cells and matrix both directly and indirectly, and via both biomechanical and biochemical means.

To investigate the mechanisms of interstitial flowinduced cell remodeling, we first tested whether interstitial flow induces similar cellular morphological responses in different fibroblast and matrix types. We compared human fibroblasts of different origin, dermal (HDFs) and lung fibroblasts (HLFs), seeded in collagen and fibrin hydrogels of similar concentrations and mechanical properties. We quantified alignment using an image analysis method based on Fast Fourier Transform (FFT) from 3D confocal stacks for cell alignment and confocal reflection images for matrix alignment in cell-free as well as cellpopulated gels. We also performed time-course studies to track the remodeling changes in acellular and fibroblastpopulated collagen matrices. Our findings help elucidate the effects of interstitial flow on cells in 3D matrices, important both for tissue remodeling when fluid flux is increased (e.g., wound healing, inflammation, and acute edema) as well as 
for tissue engineering applications where perfusion through 3D systems is involved.

\section{METHODS}

\section{Cell Culture of Human Neonatal Dermal Fibroblasts and Fetal Lung Fibroblasts}

CCD1079sk human neonatal dermal fibroblasts (HDF) and IMR-90 human fetal lung fibroblasts (HLF) from American Type Culture Collection (Manassa, VA) were cultured as previously described. ${ }^{4,23}$ They were cultured in $\alpha$-MEM supplemented with $10 \%$ fetal bovine serum (GIBCO BRL, Grand Island, NY) and 1\% penicillin/streptomycin (Sigma, St. Louis, MO). HDFs of passages 7-9 and HLFs at passages 13-14 were used in all experiments.

\section{Preparation of Interstitial Radial Flow Tissue Culture Chamber}

Cells were suspended in $2 \mathrm{mg} / \mathrm{ml}$ collagen or fibrin gel at $5 \times 10^{5}$ cells $/ \mathrm{ml}$. The collagen was prepared from rat tail tendon as previously described ${ }^{23}$ and the fibrin solution $\left(2 \mathrm{mg} / \mathrm{ml}\right.$ bovine fibrinogen, $10 \mathrm{mM} \mathrm{Ca}{ }^{2+}, 0.8 \mathrm{U} / \mathrm{ml}$ bovine thrombin, and $100 \mathrm{KIU} / \mathrm{ml}$ aprotinin) was prepared as previously described; ${ }^{28}$ all reagents were from Sigma. The cell-gel solution was pipetted into a radial interstitial flow chamber recently described, ${ }^{21-23}$ immersed in medium, and placed in a $37^{\circ} \mathrm{C} / 5 \% \mathrm{CO}_{2}$ incubator overnight. To induce interstitial flow, the chamber was connected to a sterile media reservoir via a peristaltic pump and a pressure manometer. Normal growth media was delivered at $0.012 \mathrm{ml} / \mathrm{min}$ leading to an average velocity of $12.6 \mu \mathrm{m} / \mathrm{s}$ at the inlet and $3.6 \mu \mathrm{m} / \mathrm{s}$ at the outlet. A static chamber used as a control was set up exactly as the experimental flow chamber, except that it was not connected to the flow delivery apparatus. In both groups, medium surrounded the chamber and could diffuse through the outer and inner PE rings. All cultures were maintained in a humidified $37^{\circ} \mathrm{C}, 5 \% \mathrm{CO}_{2}$ incubator for the duration of the experiment.

\section{Preparation and Imaging of Tissue Cultures for Morphological Analysis}

Alexa Fluor 488 phalloidin (Molecular Probes, Eugene, OR) and confocal reflection contrast microscopy was used to identify and examine the organization of the cells and matrix fibers, respectively. The entire chamber was first fixed by immersion in $2 \%$ paraformaldehyde in PBS for 30 min, rinsed with PBS, and permeabilized in $0.5 \%$ Triton for $30 \mathrm{~min}$. The chamber was then immersed overnight in a solution containing $132 \mathrm{nM}$ phalloidin at $4^{\circ} \mathrm{C}$. The gels were rinsed with PBS and images were taken with a laser scanning confocal microscope (LCS SP2, Leica, Mannheim, Germany).

Confocal reflection contrast microscopy was performed to visualize unstained matrix fibers using a $40 \times(1.25$
NA) oil objective lens as described earlier.,13,21 Briefly, $488 \mathrm{~nm}$ light (Ar laser) is introduced into the sample, and (i) reflected signals from both ECM and cytoskeletal fibers and (ii) emission signals from the phalloidin staining are passed through a RT30/70 filter and collected in separate photomultipliers. The samples were vertically scanned in the $z$ direction at a scanning depth of $10-30 \mu \mathrm{m}$ from the bottom coverslip. The sequential images were collected and reconstructed as 2-D projections using Leica LCS software.

\section{Image Analysis for Quantification of Alignment and Peak Orientation}

Our image analysis procedure is schematized in Fig. 1. First, we used an FFT analysis to evaluate the orientation of cells or matrix fibers in the confocal images. This indirect method transforms the original intensity image into a frequency spectrum image, from which the orientation information can be extracted. ${ }^{3,24,25,27,31}$ It has been applied previously to quantify collagen fiber alignment in scanning electron microscopy and histological images of ligaments, sclerodermal lesions, and scar tissues. ${ }^{3,31}$ Here, we extended this analysis to images collected with confocal reflection microscopy. After converting the color image to grayscale using Image $(\mathrm{NIH}$, Bethesda, MD), a MATLAB (The MathWorks, Inc., Natick, MA) program, developed to perform the FFT analysis, generated an orientation intensity frequency distribution from the image. For matrix quantification of images containing cells, the cells were digitally removed with Adobe Photoshop (San Jose, CA) prior to the analysis. If there was a preferred alignment of cells or fibers in the original image, a bellshaped frequency distribution would result, with the peak indicating the direction of orientation. If the cells or fibers were randomly oriented, there would be no pattern in the histogram, and the peak intensity would be at a random angle and would thus have no meaning.

To summarize the histograms, we defined an alignment index and an orientation angle for each image. The alignment index indicates the degree of anisotropy of the cells or the matrix through comparing the fraction of intensities within $20^{\circ}$ of the peak angle to the same fraction in a random (flat) histogram. As such, an alignment index of unity indicates an ideally random distribution and the higher the index from unity, the more dominant the alignment.

The orientation angle is the angle of the highest orientation intensity represented with respect to the flow axis. Ideal cases of perpendicular and horizontal orientations would give orientation angles of $90^{\circ}$ and $0^{\circ}$ respectively. Thus, a high alignment index with a small SD of the orientation angle indicates aligned cells or fibers with consistent orientation, whereas a low alignment index and a large SD indicates little alignment and random orientation. There is also the possibility of a low alignment index and small SD of the orientation angle, as we will see was the case with 

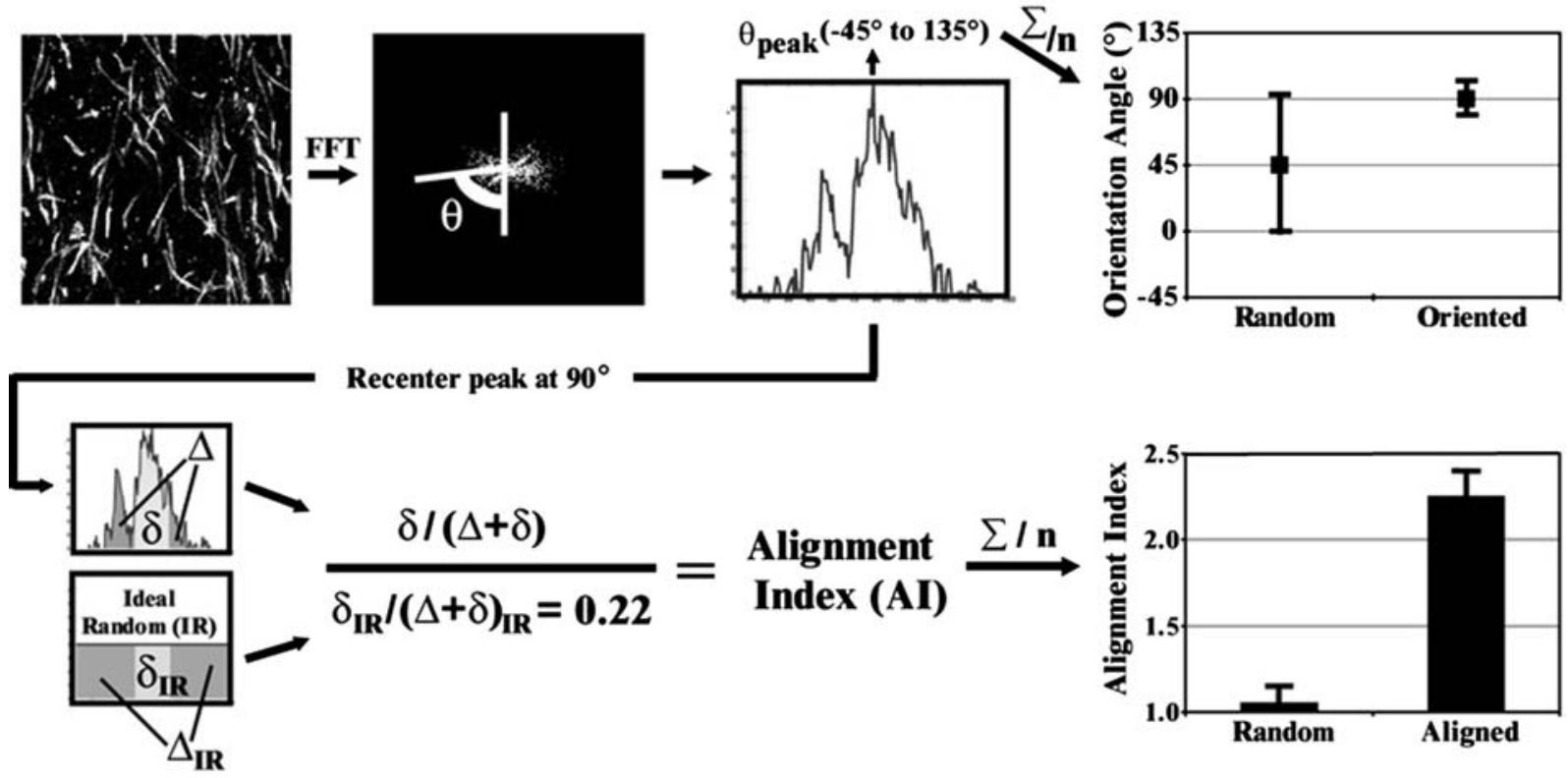

FIGURE 1. Algorithm for FFT image quantification of alignment and orientation of cells or matrix fibers. The image was imported as a matrix array before an FFT-transform into a power spectrum. The intensity at each angle was plotted to determine the orientation frequency distribution. From the distribution, the peak angle was recentered at $90^{\circ}$ before normalizing $\delta$ (sum of frequencies within $20^{\circ}$ from the peak angle) against $(\Delta+\delta)$ (total sum of all frequencies). This ratio was normalized to that of an ideally random frequency distribution $\delta_{\mathrm{IR}} /(\Delta+\delta)_{\mathrm{IR}}$ to derive the alignment index (Al). The peak orientation angle was also determined from the frequency distribution.

fibrin under flow. In this case, fibers showed very weak alignment, but nonetheless the alignment was in the same direction in all experiments. We noted that the transition from random to aligned fibers or cells occurred slowly over time.

\section{Statistical Analysis of Alignment and Orientation}

The alignment indices and peak orientation angles in the flow images were analyzed for significant differences compared to the corresponding static controls. All statistical analyses were performed using Prism 4.0 (Graphpad Software, San Diego, CA). Comparisons between two groups were assessed using unpaired Student's $t$-tests while comparisons of three groups or more were assessed using ANOVA with Tukey's post-test. The data are presented as means and standard deviations; ${ }^{*} p<0.05$ and ${ }^{* *} p<0.01$.

\section{RESULTS}

\section{Cell Alignment}

Similar to the HDFs as previously established, ${ }^{23}$ HLFs suspended in 3D collagen matrices aligned perpendicular to the direction of flow in the higher velocity region of the gel (Fig. 2). The orientation angles of HDFs and HLFs relative to the flow direction were $83.3^{\circ} \pm 18.0^{\circ}$ and $74.3^{\circ} \pm 30.5^{\circ}$, respectively. In static controls, the large $\mathrm{SD}$ in orientation angles $\left(46.3^{\circ}\right.$ and $55.7^{\circ}$ for HDFs and HLFs) coupled with low alignment indices suggest random orientation and no alignment.

The radial geometry of the flow chamber allowed us to organize the data into two regions, one closer to the inlet where velocity ranged from 12.6 to $5.6 \mu \mathrm{m} / \mathrm{s}$ (weighted average $=7.4 \mu \mathrm{m} / \mathrm{s}$ ), and the other closer to the outlet with a velocity range of $5.6-3.6 \mu \mathrm{m} / \mathrm{s}$ (average $=4.9 \mu \mathrm{m} / \mathrm{s}$ ). Corresponding shear stresses were estimated ${ }^{32}$ as $0.3-$ $0.13 \mathrm{dyn} / \mathrm{cm}^{2}$ in the inlet region and $0.13-0.09 \mathrm{dyn} / \mathrm{cm}^{2}$ in the outlet region. We found that significant alignment only occurred in the higher velocity region [Fig. 2(b) and (c)]. Furthermore, trials with higher velocities resulted in significant cell rounding and death (data not shown); thus, this velocity range of $12.6-5.6 \mu \mathrm{m} / \mathrm{s}$ was found to be optimal for cell alignment.

In contrast, when HDFs were suspended in fibrin gels of similar concentration $(2 \mathrm{mg} / \mathrm{ml})$ and subjected to interstitial flow, no significant fibroblast alignment was seen within $48 \mathrm{~h}$ [Fig. 3(a)]. The alignment indices of HDFs in fibrin gels under flow and static conditions [Fig. 3(b)] were not significantly different from each other as well as from the static controls in collagen gels.

\section{Matrix Alignment}

Using confocal reflection microscopy, we found that collagen fibers aligned perpendicular to flow in fibroblastpopulated gels after $48 \mathrm{~h}$ (Fig. 4), as shown by confocal 
DERMAL

static
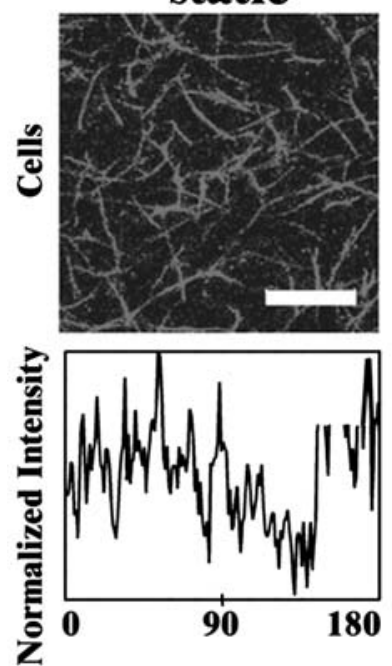

b

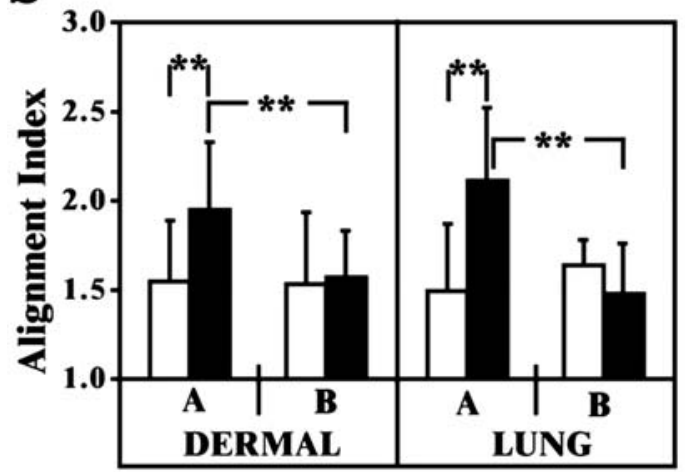

flow
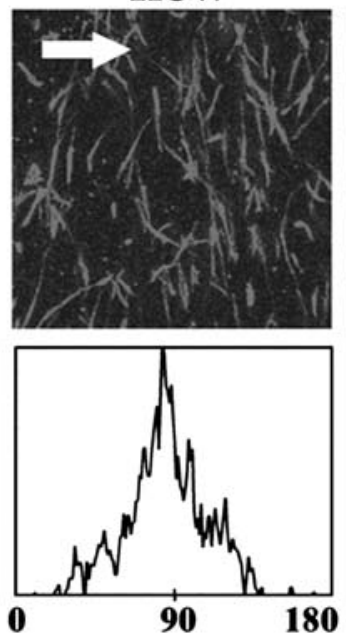

static
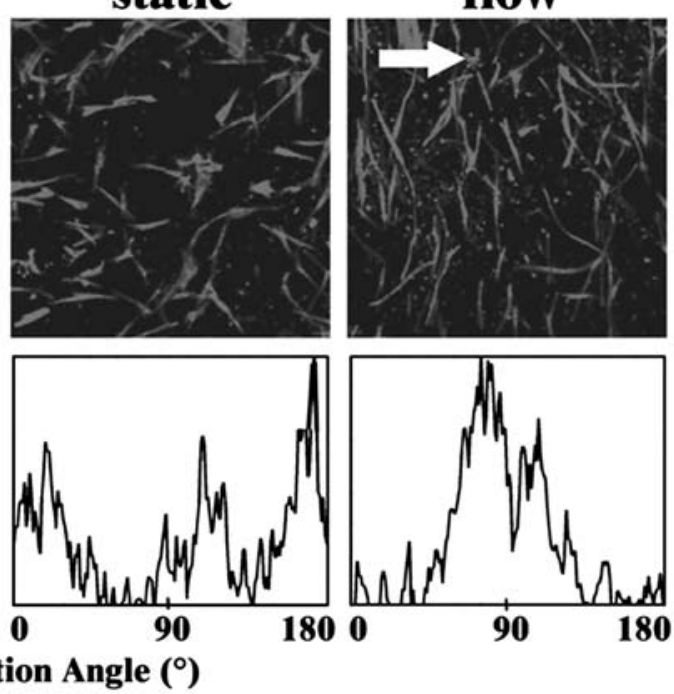

\section{flow}

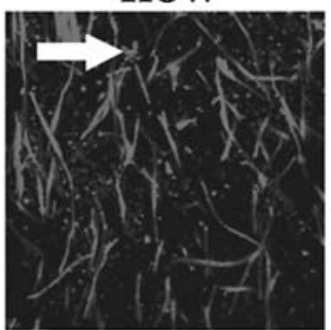

C

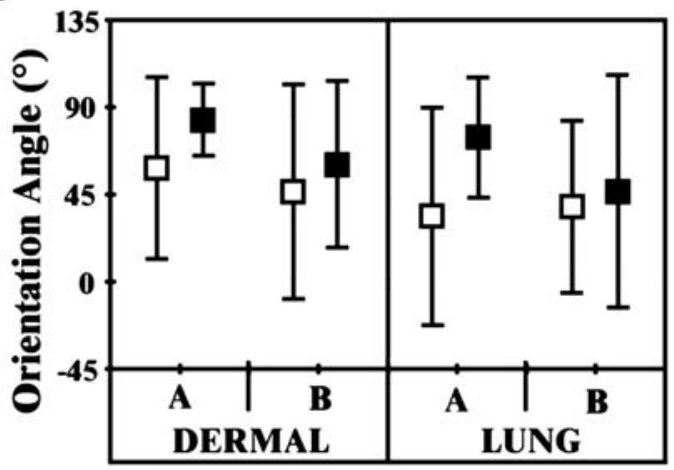

$$
\begin{gathered}
\text { 口Static } \square \text { Flow } \\
\text { A: } v=12.6-5.6 \mu \mathrm{m} / \mathrm{s} ; \tau=0.30-0.13 \mathrm{dyn} / \mathrm{cm}^{2} \\
\text { B: } \mathbf{v}=\mathbf{5 . 6 - 3 . 6} \mu \mathrm{m} / \mathrm{s} ; \tau=\mathbf{0 . 1 3 - 0 . 0 9} \mathrm{dyn} / \mathrm{cm}^{2}
\end{gathered}
$$

FIGURE 2. Interstitial flow induces the alignment of human lung as well as dermal fibroblasts (HLFs and HDFs). (a) Confocal images and orientation distributions of HLFs and HDFs in collagen matrices after $48 \mathrm{~h}$ of fluid flow (average velocity $=7.4 \mu \mathrm{m} / \mathrm{s}$ ). Bar $=$ $200 \mu \mathrm{m}$; arrow shows the direction of flow. (b) Alignment index and (c) orientation angle of HDFs and HLFs subject to average flow velocities of $7.4 \mu \mathrm{m} / \mathrm{s}$ and $4.9 \mu \mathrm{m} / \mathrm{s}$ along with static controls.

images and their respective orientation intensity distributions. Surprisingly, weak alignment in the same direction also occurred in acellular gels under flow. However, the extent of anisotropy (as reflected by alignment indices) in the acellular gels was less than that seen in cell-populated gels, indicating that although some alignment due to flow is passive, the fibroblasts also play an active role in further aligning the collagen fibers. In contrast, collagen fibers in both acellular and cell-populated static controls were not aligned; their orientation intensity histograms showed random distributions with no distinct peaks.

In fibrin, the alignment indices of matrix fibers under flow conditions were not significantly different from the static controls. However, there appeared to be an orientation tendency in the direction of flow, since the SD of the orientation angle was small. Thus, fibrin gels may weakly align parallel to flow, and although an insignificant fraction of fibers aligned, the direction of this alignment was consistent across all experiments.

We further quantified matrix alignment and orientation in HLF-populated collagen gels and compared them to HDF-populated collagen matrices (data not shown). We also found that the collagen fibers in those gels were also aligned perpendicular to flow but this alignment was weaker than what we observed for HDFs. Likewise, the fibers in static HLF controls showed no significant alignment. 

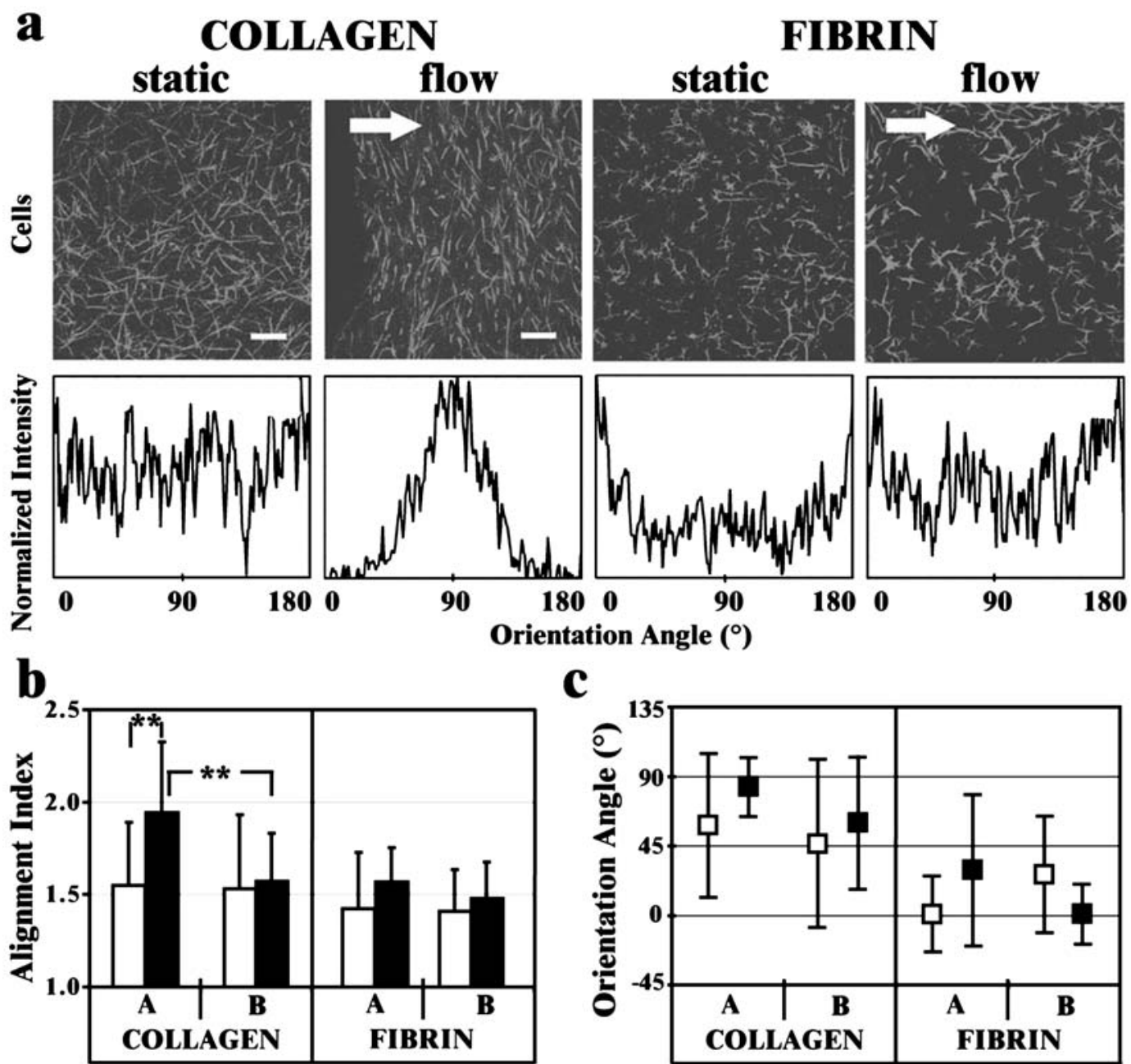

C
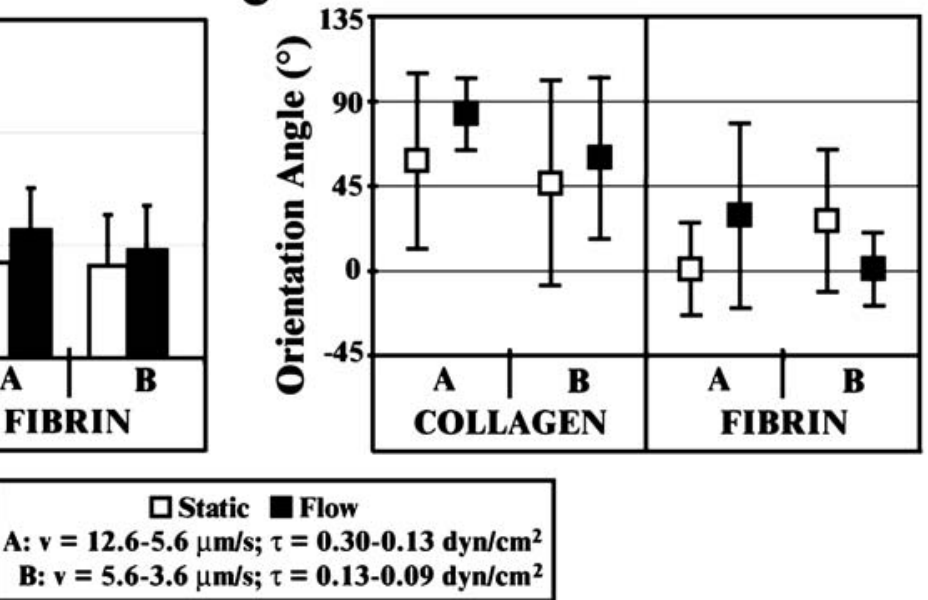

FIGURE 3. Cell alignment occurs in collagen, but not fibrin, matrices, and only at higher velocities (12.6-5.6 $\mu \mathrm{m} / \mathrm{s})$. (a) Confocal images and orientation distributions of HDFs in high velocity regions of collagen and fibrin matrices after $48 \mathrm{~h}$ of fluid flow (average velocity $=7.4 \mu \mathrm{m} / \mathrm{s}$ ). Bar $=200 \mu \mathrm{m}$; arrow shows the direction of flow. (b) Alignment index and (c) orientation angles of HDFs in high (average $=7.4 \mu \mathrm{m} / \mathrm{s}$ ) vs. low (average $=4.9 \mu \mathrm{m} / \mathrm{s}$ ) velocity regions of the cultures in collagen vs. fibrin matrices, after $48 \mathrm{~h}$.

\section{Time Course of Alignment}

To determine whether the alignment of fibroblasts occurred before, concurrent with, or after the alignment of collagen fibers, we evaluated HDF and matrix alignment at $1,3,6,12$, and $48 \mathrm{~h}$ after the onset of flow (Fig. 5) in the velocity range of $12.6-5.6 \mu \mathrm{m} / \mathrm{s}$ and found that HDF in collagen matrices became progressively aligned and perpendicularly oriented under IFF over this time frame. Beyond this time, the extent of alignment continued to increase but no changes were seen in the orientation angle of the cells (data not shown). Matrix fibers also became progressively aligned over $48 \mathrm{~h}$, as indicated by the increasing alignment indices, and were increasingly oriented in the direction perpendicular to flow (indicated by the orientation angle reaching $90^{\circ}$ with decreasing SD). Furthermore, the cell-populated matrices aligned more substantially at 12 and $48 \mathrm{~h}$ than the acellular matrices, suggesting an active role of the cells in matrix alignment.

Thus, in comparing the time course of cell vs. matrix alignment, we see that cell alignment lagged behind that of matrix fibers. On the other hand, the alignment by matrix fibers is more extensive when cells are present as compared to when cells are not present. These data suggest that both passive and active reorganization mechanisms act in concert. 
$\mathbf{a}$

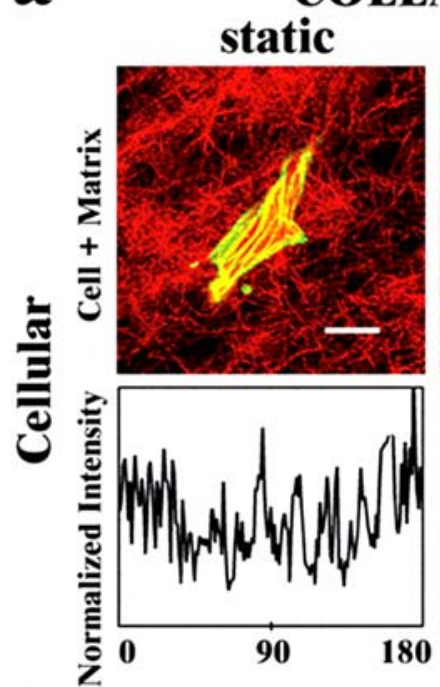

b
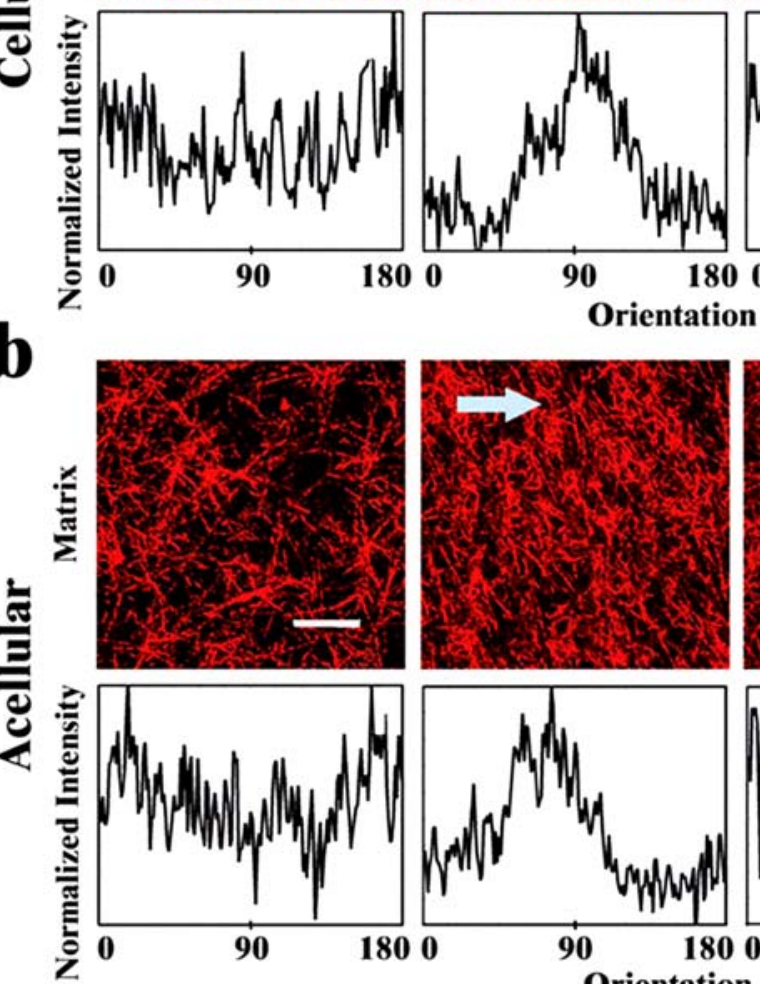

Orientation Angle ( ${ }^{\circ}$ )

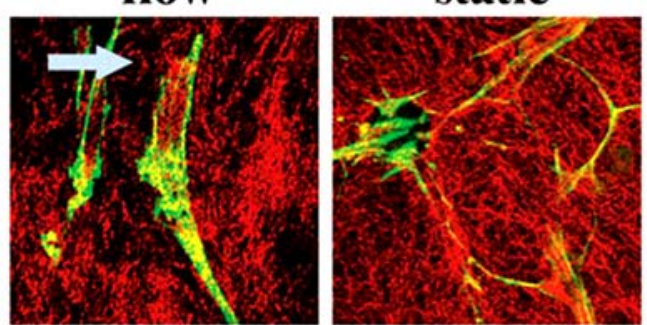

FIBRIN

static

\section{flow}
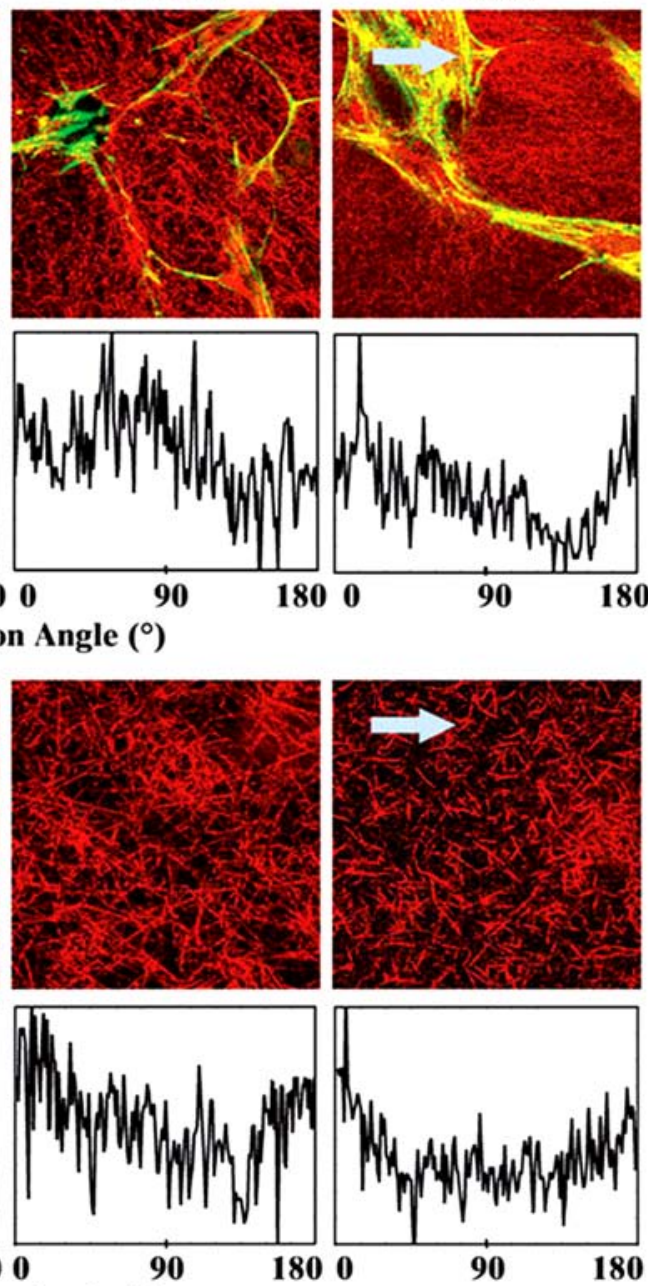

Orientation Angle $\left({ }^{\circ}\right)$
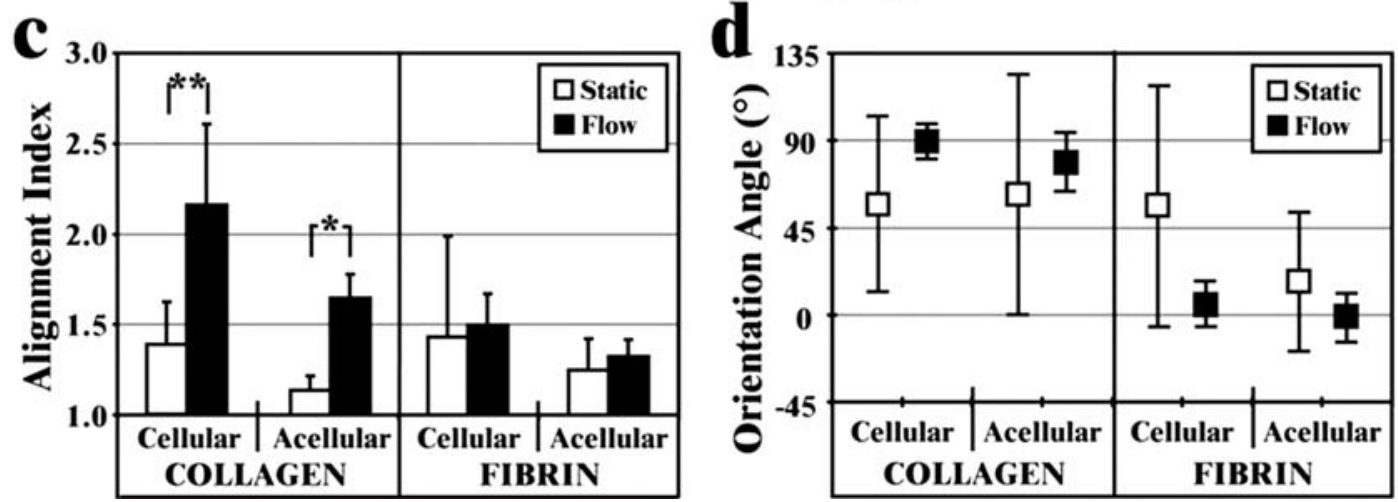

FIGURE 4. Perpendicular matrix alignment occurs in collagen but not fibrin matrices, and even occurs to a small extent in the absence of cells. Representative confocal reflection images and their orientation intensity distributions at $48 \mathrm{~h}$ of (a) cell-populated and (b) acellular matrices. In cell-populated gels, the cells were digitally removed from the images before the FFT algorithm was applied to ensure that only the matrix reflectance was quantified. Bar $=20 \mu \mathrm{m}$; arrow indicates the direction of flow. (c) Alignment index and (d) orientation angle of collagen and fibrin fibers in acellular vs. fibroblast-populated matrices. 
d
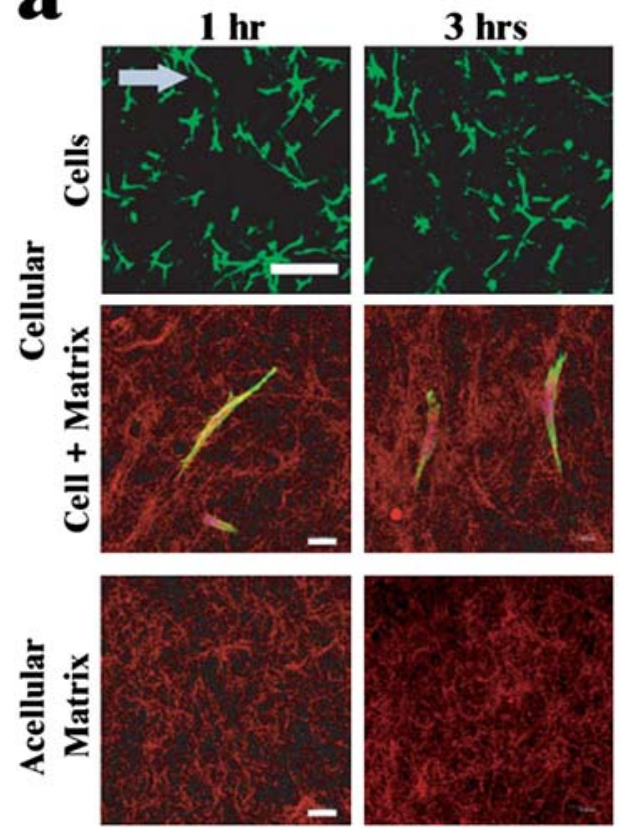

b

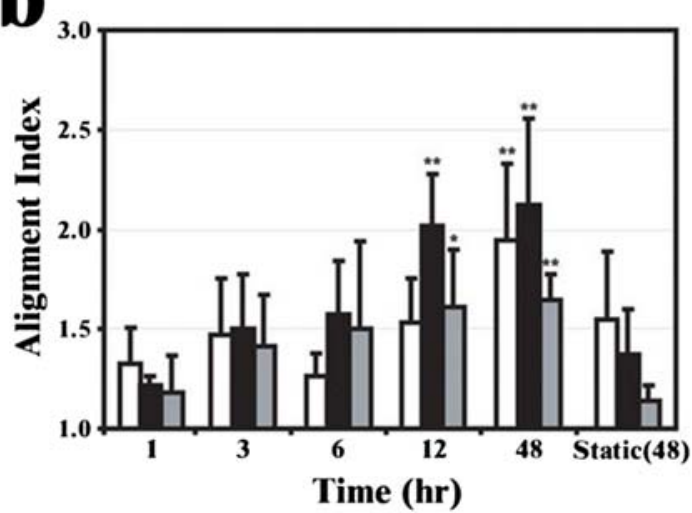

6 hrs
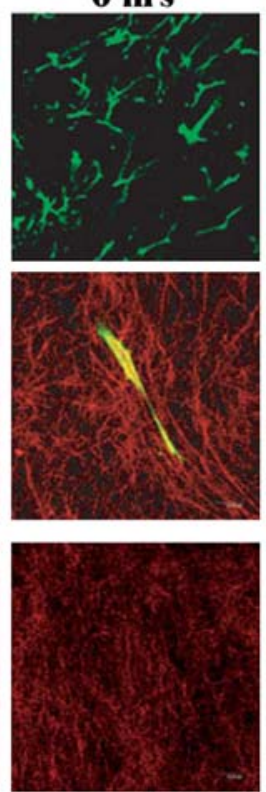

$12 \mathrm{hrs}$
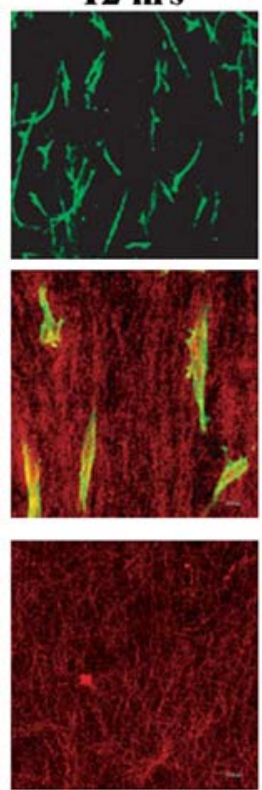

48 hrs
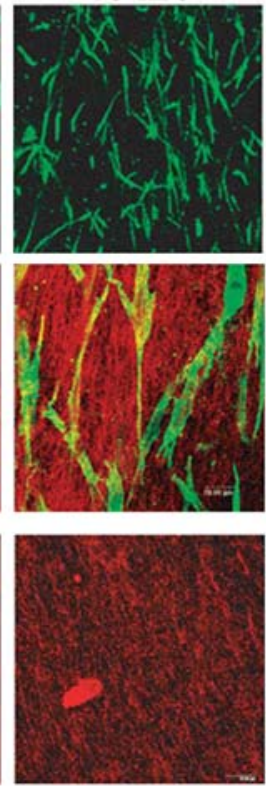

c

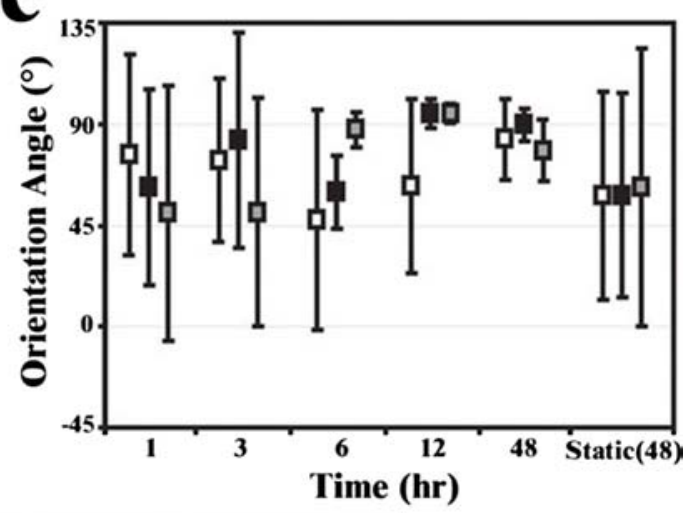

$\square$ Cell Matrix (Cellular) $\square$ Matrix (Acellular)

FIGURE 5. Interstitial flow remodels matrix architecture within $12 \mathrm{~h}$ and induces cell alignment within $48 \mathrm{~h}$. (a) Confocal images of fibroblasts in collagen matrices, and confocal reflection images of cellular and acellular collagen matrices taken at 1, 3, 6, 12 and $48 \mathrm{~h}$. Confocal and confocal reflection images were 2D projections of $100 \mu \mathrm{m}$ and $5 \mu \mathrm{m}$ optical sections of the gel, respectively at regions subjected to average flow velocity of $7.4 \mu \mathrm{m} / \mathrm{s}$. Bar $=200 \mu \mathrm{m}$ and $20 \mu \mathrm{m}$ for confocal and confocal reflection images, respectively; arrow shows the direction of flow. (b) Alignment index and (c) orientation angle of cells and collagen fibers over a time course of $48 \mathrm{~h}$.

\section{DISCUSSION}

We previously demonstrated that low levels of interstitial flow cause fibroblasts within a 3D collagen matrix to align perpendicular to flow. Here, we explored mechanisms of this alignment and demonstrated that both passive and active reorganization mechanisms are involved in the interstitial flow-induced cell and matrix alignment. In addition, the data suggest the following mechanism of remodeling: interstitial flow initially induces weak matrix anisotropy in the perpendicular direction to flow, which provides contact guidance for initiating cell alignment; the cells then continue to align the ECM and themselves under interstitial flow. The alignment occurred on a timescale of 6-12 $\mathrm{h}$ and in the velocity range of $12.6-5.6 \mu \mathrm{m} / \mathrm{s}$. It occurred in both human lung and dermal fibroblasts.

Since fibroblasts align under interstitial flow in collagen but not fibrin matrices, it is probable that the matrix architecture strongly influences how interstitial flow affects cells in a 3D environment. Collagens are the most common fibrous proteins in the human body, and the reconstituted type I collagen gel used here is a highly hydrated (>99\%) 
network of entangled, but not covalently crosslinked, collagen fibrils. ${ }^{26}$ In contrast, fibrin is the primary structural component of blood clots; they are highly cross-linked and cannot be reorganized by the cells but rather only proteolytically degraded. Thus, while cells can easily move around in collagen matrices and reorganize the ECM fibers without degrading the matrix,${ }^{12,14}$ fibrin is a temporary matrix designed to degrade rather than reorganize, and this is probably why very little cell and matrix alignment was seen with flow through fibrin matrices.

Furthermore, we recently found that $\alpha_{1} \beta_{1}$ integrin plays a critical role in flow-induced cell and collagen alignment. ${ }^{22}$ In contrast, $\alpha_{2} \beta_{1}$ integrins have been shown to be important in collagen gel contraction by fibroblasts ${ }^{19}$ (and we confirmed that $\alpha_{1} \beta_{1}$ integrin was not required for gel con$\operatorname{traction}^{22}$ ). Together with our findings that weak matrix alignment passively occurs prior to cell alignment, these data suggest that interstitial flow-induced alignment may not involve cell contraction but instead result mainly from passive forces.

The acellular alignment may be considered somewhat surprising since, from a purely mechanical point of view, a parallel fiber alignment would reduce fluid drag through the network and lead to a higher hydraulic conductivity than that of a perpendicular organization, ${ }^{18}$ and fiber extrusion processes generally tend to yield aligned fibers parallel to flow. However, it is important to note that in our system (i) the collagen fibers are rigid and highly entangled in a random position during gelation, before flow onset rather than under flow during gelation, and (ii) the initially random fiber orientation will lead to a complex flow field that may induce a complex force distribution on the array, tending to compact the network rather than align it if mechanically hindered.

Although our studies were done in vitro in reconstituted collagen gel networks, there are potential implications for this alignment phenomenon in vivo. Collagen alignment contributes to the mechanical strength of tissues and therefore plays an important role in the optimal functioning of many tissues. In addition, alignment is generally observed in differentiated tissues such as scar tissues in wounds (both normal and fibrotic) ${ }^{10,29}$ and stromal response in desmoplastic tumors. ${ }^{6,7}$ Fibroblasts are a major mediator of the wound healing process, ${ }^{9,16}$ where they differentiate into myofibroblasts and acquire smooth muscle features. Interstitial flow is likely increased during wound healing, when remodeling blood vessels, infiltrating inflammatory cells, and tissue proteolysis all increase fluid flux through the ECM; indeed, we recently showed that interstitial flow alone is sufficient to induce fibroblast-to-myofibroblast differentiation in vitro.

Our findings are also relevant to tissue engineering. Culturing fibroblast-populated collagen gels under various types of mechanical loading such as tension and compaction has been used to improve mechanical properties and induce alignment, ${ }^{5,8,15,30,34}$ and other methods like applying magnetic fields can also align collagen matrices. ${ }^{1,17}$ Here we show that low levels of interstitial flow may also be used to align a collagen matrix and thereby alter its mechanical properties.

In conclusion, this study highlights the interplay between interstitial fluid forces, cells, and the extracellular matrix. It demonstrates how interstitial fluid flow may contribute to cell alignment in tissues through both passive effects on the matrix fibers and active effects on the cells, and has relevance for understanding physiological and pathological tissue remodeling as well as potential application in tissue engineering.

\section{ACKNOWLEDGMENTS}

This work was funded by grants from the Whitaker Foundation (RG-01-0348) and the National Science Foundation (BES-0134551).

\section{REFERENCES}

${ }^{1}$ Barocas, V. H., T. S. Girton, and R. T. Tranquillo. Engineered alignment in media equivalents: Magnetic prealignment and mandrel compaction. J. Biomech. Eng. 120:660-666, 1998.

${ }^{2}$ Brightman, A. O., B. P. Rajwa, J. E. Sturgis, M. E. McCallister, J. P. Robinson, and S. L. Voytik-Harbin. Time-lapse confocal reflection microscopy of collagen fibrillogenesis and extracellular matrix assembly in vitro. Biopolymers 54:222-234, 2000.

${ }^{3}$ Chaudhuri, S., H. Nguyen, R. M. Rangayyan, S. Walsh, and C. B. Frank. A Fourier domain directional filtering method for analysis of collagen alignment in ligaments. IEEE Trans. Biomed. Eng. 34:509-518, 1987.

${ }^{4}$ Choe, M. M., P. H. S. Sporn, and M. A. Swartz. An in vitro airway wall model of remodeling. Am. J. Physiol. Lung Cell Mol. Physiol. 285:L427-L433, 2003.

${ }^{5}$ Costa, K. D., E. J. Lee, and J. W. Holmes. Creating alignment and anisotropy in engineered heart tissue: Role of boundary conditions in a model three-dimensional culture system. Tissue Eng. 9:567-577, 2003.

${ }^{6}$ De Wever, O., and M. Mareel. Role of tissue stroma in cancer cell invasion. J. Pathol. 200:429-447, 2003.

${ }^{7}$ Desmouliere, A., C. Guyot, and C. Gabbiani. The stroma reaction myofibroblast: A key player in the control of tumor cell behavior. Int. J. Dev. Biol. 48:509-517, 2004.

${ }^{8}$ Eastwood, M., V. C. Mudera, D. A. McGrouther, and R. A. Brown. Effect of precise mechanical loading on fibroblast populated collagen lattices: Morphological changes. Cell Motil. Cytoskeleton 40:13-21, 1998.

${ }^{9}$ Eckes, B., P. Zigrino, D. Kessler, O. Holtkotter, P. Shephard, C. Mauch, and T. Krieg. Fibroblast-matrix interactions in wound healing and fibrosis. Matrix Biol. 19:325-332, 2000.

${ }^{10}$ Ehrlich, H. P., and T. M. Krummel. Regulation of wound healing from a connective tissue perspective. Wound Repair Regen. 4:203-210, 1996.

${ }^{11}$ Farsi, J. M., and J. E. Aubin. Microfilament rearrangements during fibroblast-induced contraction of three-dimensional hydrated collagen gels. Cell Motil. 4:29-40, 1984.

${ }^{12}$ Friedl, P., and E. B. Brocker. The biology of cell locomotion within three-dimensional extracellular matrix. Cell. Mol. Life Sci. 57:41-64, 2000. 
${ }^{13}$ Friedl, P., K. Maaser, C. E. Klein, B. Niggemann, G. Krohne, and K. S. Zanker. Migration of highly aggressive mv3 melanoma cells in 3-dimensional collagen lattices results in local matrix reorganization and shedding of alpha2 and beta1 integrins and CD44. Cancer Res. 57:2061-2070, 1997.

${ }^{14}$ Friedl, P., K. S. Zanker, and E. B. Brocker. Cell migration strategies in 3-d extracellular matrix: Differences in morphology, cell matrix interactions, and integrin function. Microsc. Res. Technol. 43:369-378, 1998.

${ }^{15}$ Girton, T. S., V. H. Barocas, and R. T. Tranquillo. Confined compression of a tissue equivalent: Collagen fibril and cell alignment in response to anisotropic strain. J. Biomech. Eng. 124:568-575, 2002.

${ }^{16}$ Grinnell, F. Fibroblasts, myofibroblasts, and wound contraction. J. Cell Biol. 124:401-404, 1994.

${ }^{17}$ Guido, S., and R. T. Tranquillo. A methodology for the systematic and quantitative study of cell contact guidance in oriented collagen gels. Correlation of fibroblast orientation and gel birefringence. J. Cell Sci. 105:317-31, 1993.

${ }^{18}$ Happel, J., and H. Brenner. Low Reynolds Number Hydrodynamics. Dordrecht: Kluwer Academic Publishers, 1991, pp. 392-399.

${ }^{19}$ Jenkins, G., K. L. Redwood, L. Meadows, and M. R. Green. Effect of gel re-organization and tensional forces on alpha 2 beta 1 integrin levels in dermal fibroblasts. Eur. J. Biochem. 263:93-103, 1999.

${ }^{20}$ Lee, A. A., D. A. Graham, S. Dela Cruz, A. Ratcliffe, and W. J. Karlon. Fluid shear stress-induced alignment of cultured vascular smooth muscle cells. J. Biomech. Eng. 124:37-43, 2002.

${ }^{21} \mathrm{Ng}$, C. P., C. L. E. Helm, and M. A. Swartz. Interstitial flow differentially stimulates blood and lymphatic endothelial cell morphogenesis in vitro. Microvasc. Res. 68:258-264, 2004.

${ }^{22} \mathrm{Ng}$, C. P., B. Hinz, and M. A. Swartz. Interstitial fluid flow induces myofibroblast differentiation and collagen alignment in vitro. J. Cell Sci. 118:4731-4739, 2005.
${ }^{23} \mathrm{Ng}$, C. P., and M. A. Swartz. Fibroblast alignment under interstitial fluid flow using a novel 3-d tissue culture model. Am. J. Physiol. Heart Circ. Physiol. 284:H1771-H1777, 2003.

${ }^{24}$ Nishimura, T., and M. P. Ansell. Fast Fourier transform and filtered image analyses of fiber orientation in osb. Wood Sci. Technol. 36:287-307, 2002.

${ }^{25}$ Palmer, B. M., and R. Bizios. Quantitative characterization of vascular endothelial cell morphology and orientation using Fourier transform analysis. J. Biomech. Eng. 119:159$165,1997$.

${ }^{26}$ Pedersen, J. A., and M. A. Swartz. Mechanobiology in the third dimension. Ann. Biomed. Eng.33:1469-1490, 2005.

${ }^{27}$ Pourdeyhimi, B., R. Dent, and H. Davis. Measuring fiber orientation in nonwovens, 3: Fourier transform. Text. Res. J. 67:143$151,1997$.

${ }^{28}$ Schense, J. C., and J. A. Hubbell. Cross-linking exogenous bifunctional peptides into fibrin gels with factor xiiia. Bioconjug. Chem. 10:75-81, 1999.

${ }^{29}$ Tomasek, J. J., G. Gabbiani, B. Hinz, C. Chaponnier, and R. A. Brown. Myofibroblasts and mechano-regulation of connective tissue remodelling. Nat. Rev. Mol. Cell Biol. 3:349-363, 2002.

${ }^{30}$ Tranquillo, R. T. Self-organization of tissue-equivalents: The nature and role of contact guidance. Biochem. Soc. Symp. 65:2742, 1999.

${ }^{31}$ van Zuijlen, P. P. M., H. J. C. de Vries, E. N. Lamme, J. S. E. Coppens, J. van Marle, R. W. Kreis, and E. Middelkoop. Morphometry of dermal collagen orientation by fourier analysis is superior to multi-observer assessment. J. Pathol. 198:284 291, 2002.

${ }^{32}$ Wang, D. M., and J. M. Tarbell. Modeling interstitial flow in an artery wall allows estimation of wall shear stress on smooth muscle cells. J. Biomech. Eng. 117:358-63, 1995.

${ }^{33}$ Wang, J. H., and E. S. Grood. The strain magnitude and contact guidance determine orientation response of fibroblasts to cyclic substrate strains. Connect. Tissue Res. 41:29-36, 2000.

${ }^{34}$ Wei, J., and M. B. Russ. Convection and diffusion in tissues and tissue-cultures. J. Theor. Biol. 66:775-787, 1977. 